Classification

Physics Abstracts

$07.80-82.80-82.70 \mathrm{Kj}$

\title{
EELS-ESI Identification of Heterogeneous Suspensions of Aquatic Microparticles
}

\author{
Didier Perret $\left({ }^{*}\right)$, Charles-Philippe Lienemann and Denis Mavrocordatos \\ Institut de Chimie Minérale et Analytique, Université de Lausanne, $\mathrm{BCH}, \mathrm{CH}-1015$ Lausanne, \\ Switzerland
}

(Received September 19; accepted December 16, 1994)

\begin{abstract}
Submicron mineral particles in freshwaters represent an important proportion of the total particle number. These microparticles are frequently associated to macromolecular organic matter and have a high specific surface area; they might thus be strong heavy metal and nutrient scavengers in aquatic systems. Their non-artefacted characterization at the level of individual particles represents a promising challenge, while preservation of the undisturbed state of natural fragile specimens is a prerequisite. We have used a TEM-EELS-ESI procedure which allows the identification of mineral microparticles in individual model suspensions of iron oxides, aluminum oxides and clays in the presence of polysaccharides, at concentrations typical of natural levels. As expected, core-loss ionization edge intensities measured by this method are limited by the thickness of particles. Heterogeneous mixtures of the former microparticles/macromolecules were prepared in order to mimic natural specimens. Under optimum analytical conditions, it was possible to extract the very nature of these entities, to reveal low electron-density polysaccharides and even to identify microparticles masked within complex aggregates.
\end{abstract}

\section{Introduction}

Mineral particles (iron (oxy)hydroxides, silica, aluminum and manganese oxides, clays) have been evidenced to play a key role in the transport and distribution of trace heavy metals and anthropogenic organic pollutants within natural aquatic systems [1, 2].

However, due to high sample heterogeneity (particle sizes, morphologies and types) and analytical limitations (negligible mass concentration), little is known on the speciation of submicron colloids. These microparticles are supposed to act as the major scavengers of pollutants because of their large specific surface area, their frequent association to macromolecular organic networks of aquogenic (polysaccharides) or pedogenic (humic and fulvic acids) origin and their high number concentration (Fig. 1) [3-7].

$\left({ }^{*}\right)$ To whom correspondence should be addressed. 


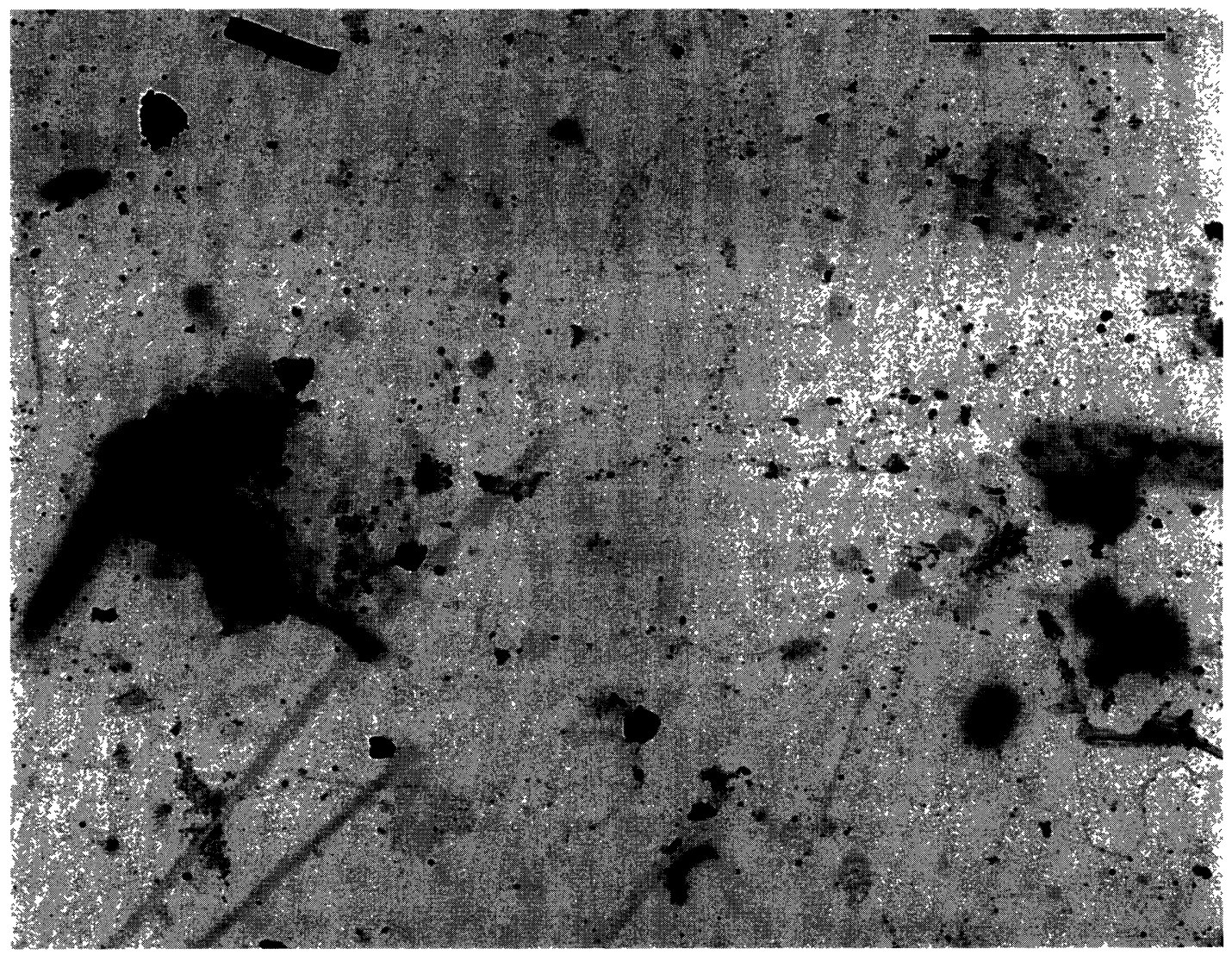

Fig. 1. - Bright field TEM of a lacustrine sample (Lake Lugano, Switzerland) showing the usual high heterogeneity of particle types and sizes, and the numerous mineral microparticles/organic fibrils associations. Bar $=2 \mu \mathrm{m}$.

In order to clearly understand, modelize and predict the transport behaviour of pollutants in natural waters, it is nowadays necessary to determine the physico-chemical characteristics and the reactivity of individual mineral microparticles and associated organic macromolecules at a high spatial resolution.

Analytical Electron Microscopy in SEM-EDS or STEM-EDS mode has proved to be an efficient technique for analyzing natural aquatic samples at the micrometer or submicrometer level [8-12], but usually fails in characterizing particles in the $<100 \mathrm{~nm}$ range except when they are coagulated into larger entities, thus being element-integrated over the total volume of the aggregate. Furthermore, carbon-containing particles are not detected by means of conventional (Be window) EDS, thus strongly limiting its usefulness when dealing with mineral microparticles/organic macromolecules associations.

The limitations encountered in EDS analysis of submicron particles has motivated us to experiment the capabilities of EELS methodology for the characterization of these microparticles, because EELS is constrained to very thin $(<100-200 \mathrm{~nm})$ samples, has a high sensitivity for light elements, and is capable of extracting elemental information at the nanometer level [13].

On the other hand, specific requisites are needed for a non-artefacted characterization of aquatic 
samples. Specimens cannot be prepared by classical embedding-ultramicrotomy procedures used in the biological science, because the 3-dimensional networks of organic macromolecular assemblies supporting mineral microparticles would appear as segmented sub-parts, without apparent connection, thus producing biased 2-dimensional images of the real situation. As the specimen is prepared by direct deposition of material on TEM grids, the EELS analysis will be dependent on the thickness of each individual particle, which might be limiting for microparticles in the $>150$ nm range [14].

In a previous study [15], we have demonstrated that a combination of zero-loss imaging, Contrast Tuning (CT) [16], Electron Spectroscopic Imaging (ESI) and Electron Energy Loss Spectrometry (EELS) could ascertain the existence of strong interactions between hematite microparticles and xanthan polyssaccharides forming large networks. These results were in agreement with observations in natural aquatic samples, and could be explained by the theory of bridging developed to explain the stability of particles in the presence of macromolecules $[17,18]$.

In the present study, we have used mixtures of standard mineral microparticles (hematite, aluminum oxide, kaolinite) and fibrilar high MW polysaccharides (xanthan) as a laboratory model to mimic natural waters and in order to test the applicability of EELS methods to complex heterogeneous aquatic suspensions.

An Energy Filtering Transmission Electron Microscopic approach (EF-TEM) allowed characterization of these mineral-organic mixtures. The results presented below show that CT reveals the ultrastructure of polysaccharides under non-disturbing conditions, while ESI and EELS allow the physico-chemical identification of individual mineral particles at the submicron level.

\section{EF-TEM Analysis}

The complete procedure leading to energy filtered images and energy-loss spectra has been discussed in details elsewhere $[13,16]$; it will be briefly described here.

On an EF-TEM, incident electrons with energy $E_{0}$ interacting with the specimen are, amongst other processes, scattered in the forward direction and can be selectively filtered prior to being detected, according to their energy $E_{0}-\Delta E_{\text {loss, }}$, where $\Delta E_{\text {loss }}$ represents the quantity of energy which has been lost by the incident electrons during electron-matter interactions. Recording of the intensity of the measured signal versus $\Delta E_{\text {loss }}$ (Electron Energy Loss Spectrum, EELS) over a large energy interval $(0-2000 \mathrm{eV})$ reveals 3 basic processes:

- $\Delta E_{\text {loss }}=0$ (zero-loss peak): incident electrons are unscattered or elastically scattered (within the resolution limit of the spectrometer). Filtered images of the irradiated specimen recorded at $\Delta E_{\text {loss }}=0$ (zero-loss imaging) usually show an increase in contrast.

- $0<\Delta E_{\text {loss }}<100 \mathrm{eV}$ (low-loss region): incident electrons allow collective excitation of valence electrons within the specimen. Under certain circumstances, these plasmons may reveal information on the molecular neighbouring of analyzed elements.

- $100 \mathrm{eV}<\Delta E_{\text {loss }}<2000 \mathrm{eV}$ (core-loss region): at certain discrete $\Delta E_{\text {loss }}$ values, an increase in signal is observed, which quantum-mechanically relies on the ionization of core electrons surrounding the elements present in the specimen. Imaging the latter at these specific energies allows chemical discrimination (chemical mapping) of the constituting elements, provided the background signal is stripped out of the ionization edges (Electron Spectroscopic Imaging, ESI).

In addition, the so-called structure-sensitive region below the carbon $\mathrm{K}$ ionization edge $(284 \mathrm{eV})$ can be profitably used in order to acquire filtered images (Contrast Tuning, CT) where carbon- 
containing entities are revealed with a much stronger contrast than in unfiltered bright-field images.

\section{Experimental}

3.1 Model Mineral Colloids and Polysaccharides. - Hematite was obtained by forced hydrolysis of a $3.5 \times 10^{-2} \mathrm{M}$ solution of $\mathrm{Fe}\left(\mathrm{ClO}_{4}\right)_{3}$ in $5 \times 10^{-2} \mathrm{M} \mathrm{HClO}_{4}$ (24h boiling under reflux) followed by three washing/ultracentrifugation steps into fresh $\mathrm{HClO}_{4}$ [19-21]. The resulting stock suspension $\left(2 \mathrm{~g} / \mathrm{l}\right.$, measured by ICP-AES after $24 \mathrm{~h}$ digestion at $100{ }^{\circ} \mathrm{C}$ in $5 \% \mathrm{HNO}_{3} / 0.1 \mathrm{M}$ $\mathrm{H}_{2} \mathrm{NOHxHCl}$ ) contained monodisperse (70 $\pm 5 \mathrm{~nm}$ diameter) $\alpha-\mathrm{Fe}_{2} \mathrm{O}_{3}$ (crystallinity confirmed by XRD).

Fresh monodisperse $(13 \mathrm{~nm})$ microparticles of aluminum oxide $\left(\mathrm{Al}_{2} \mathrm{O}_{3}\right.$; gift from Degussa) were suspended at $0.1 \mathrm{mg} / \mathrm{l}$ in ultrapure water (Elgastat UHQII) and stirred for $2 \mathrm{~h}$ prior to use.

Purified clay (kaolinite, $\mathrm{Al}_{2} \mathrm{Si}_{2} \mathrm{O}_{5}(\mathrm{OH})_{4}$; Wards) was suspended in water at $1 \mathrm{~g} / \mathrm{l}$ and stirred for $12 \mathrm{~h}$. A fast centrifugation $\left(\mathrm{RCF}_{\max }=5 \times 10^{4} \mathrm{~g} ; 10 \mathrm{~min}\right)$ of this crude suspension allowed elimination of aggregates and individual particules with sizes $>500 \mathrm{~nm}$; the supernatant was retained for preparation of TEM specimens.

Xanthan (MW $=2 \times 10^{6}$ dalton; repeating units of D-glucose with a side-chain composed of D- glucuronic acid and D-mannose containing an acetyl and a pyruvate group) was obtained as a biopolymer concentrated gel produced by Pseudomonas campestris (Flocon 4800C; Pfizer). Diluted suspensions of this polysaccharide (ca. $0.2 \mathrm{~g} / \mathrm{l}$ ) were freshly prepared in $10^{-3} \mathrm{M} \mathrm{HClO}_{4}$ and stirred for $24 \mathrm{~h}$ prior to use in order to avoid fibrils coalescence.

3.2 Preparation of Suspensions and Heterogeneous TEM Specimens. - Preparation of TEM specimens from natural or synthetic aquatic suspensions requires special attention in order to avoid physico-chemical transformations (coagulation, dissolution, shrinkage of fragile material) or biased size distributions [22].

Quantitatively representative and undisturbed specimens can be obtained for TEM visualization with optimum coverage of grids by microparticles, through the following scheme:

- Centrifugation conditions (relative centrifugal force, duration, volume) are determined according to the concentration, size and density of microparticles in the initial suspensions [3].

- Prior to centrifugation, a minute amount of uranyl acetate ( $\leq 0.1 \%$ final concentration) is added to the suspensions in order to mildly stain xanthan fibrils en bloc.

- Suspensions are then ultracentrifuged above TEM grids [22, 23].

Optimum EF-TEM analysis conditions (magnification, energy window, collection angle, duration) were determined with individual suspensions of hematite (identification of iron), respectively kaolinite (identification of aluminum and silicon). Then, heterogeneous specimens of the different colloids and polysaccharide were prepared from individual suspensions which were successively centrifuged on the same TEM grids ( $\mathrm{Cu}^{0} 200$ mesh, collodion- covered, carbon-coated).

A mixture of hematite $(0.1 \mathrm{mg} / \mathrm{l})$ and xanthan $(0.2 \mathrm{mg} / \mathrm{l})$ was freshly prepared (5 min stirring, $24 \mathrm{~h}$ reaction). In order to enhance their contrast under TEM, xanthan fibrils were stained with uranyl acetate. This mixture was then ultracentrifuged $\left(\mathrm{RCF}_{\max }=5 \times 10^{4} \mathrm{~g}, 15 \mathrm{~min} ; 4 \mathrm{ml}\right.$ mixture) above grids. After removal of supernatant, the suspension of aluminium oxide $(0.1 \mathrm{mg} / \mathrm{l})$ was ultracentrifuged $\left(\mathrm{RCF}_{\max }=5 \times 10^{4} \mathrm{~g}, 55 \mathrm{~min} ; 4 \mathrm{ml}\right.$ mixture) above the previous grids. The procedure was finally repeated for the kaolinite suspension $\left(\mathrm{RCF}_{\max }=5 \times 10^{4} \mathrm{~g}, 15 \mathrm{~min} ; 4 \mathrm{ml}\right.$ mixture). 
3.3 InSTRUMENTATION. - Electron microscopy observations were done on a medium resolution TEM (Zeiss EM10; $80 \mathrm{keV}$ ) and on an EF-TEM (Zeiss CEM902 operated at $80 \mathrm{KeV}$ ) equipped with a Castaing-Henry filter, in Contrast Tuning (CT), Electron Spectroscopic Imaging (ESI) and Electron Energy Loss Spectrometry (EELS) modes.

3.4 EF-TEM AnALYSis Conditions. - Figures 2, 3 and 4 have been obtained with individual suspensions of hematite or kaolinite; they show ESI and EELS of iron, aluminum and silicon under optimum conditions. The contrast of ESI images was enhanced for better visualization.

EEL Spectra are acquired on individual microparticles with an energy window of $3 \mathrm{eV}$, a scan rate of $0.5 \mathrm{eV} / \mathrm{s}$ and a collection angle of $18 \mathrm{mrad}$. They clearly exhibit $\mathrm{O} \mathrm{K}$ edge at $530 \mathrm{eV}$ and Fe $L_{3}$ and $L_{2}$ edges at 708 and $721 \mathrm{eV}$ for hematite (Fig. 2a), while Al K and Si K edges appear at $1550 \mathrm{eV}$ (maximum intensity at $1600 \mathrm{eV}$ ), respectively $1830 \mathrm{eV}$ (maximum intensity at $1850 \mathrm{eV}$ ) for kaolinite (Figs. 3a and 4a). These values are in agreement with tabulated data [24, 25].

ESI images of these individual suspensions of hematite and kaolinite are obtained by the difference method [16]: two pre-edge images are used in order to extrapolate and subtract background $\left(A \cdot E^{-r}\right)$ from the image taken at the maximum of the core-loss ionization edge.

EEL Spectra were recorded for each microparticle prior to ESI in order to check for artificial mass-thickness contrast; particles with a large thickness or density compared to their surrounding exhibited this effect and were excluded from the study.

With an energy window of $13 \mathrm{eV}, \mathrm{Fe} L_{3}$ and $L_{2}$ edges are not discriminated and show a maximum at $\Delta E_{\text {loss }}=716 \mathrm{eV}\left(\mathrm{Fe} L_{2,3}\right)$. Fe pre-edges at 680 and $690 \mathrm{eV}$ were used for background extrapolation at $716 \mathrm{eV}$. The primary magnification used for ESI of iron (30'000x) and the relatively short acquisition time (8s for each image) allow recording of elemental information over a large area (Fig. 2b), where elements other than iron are spectroscopically excluded, without inducing beam damage to these microparticles.

Elemental imaging of aluminum present in kaolinite (Fig. 3b) was obtained from the $\mathrm{Al} \mathrm{K}$ edge (energy window: $13 \mathrm{eV}$; acquisition time: $8 \mathrm{~s}$ /image); pre-edges at 1540 and $1550 \mathrm{eV}$ were used
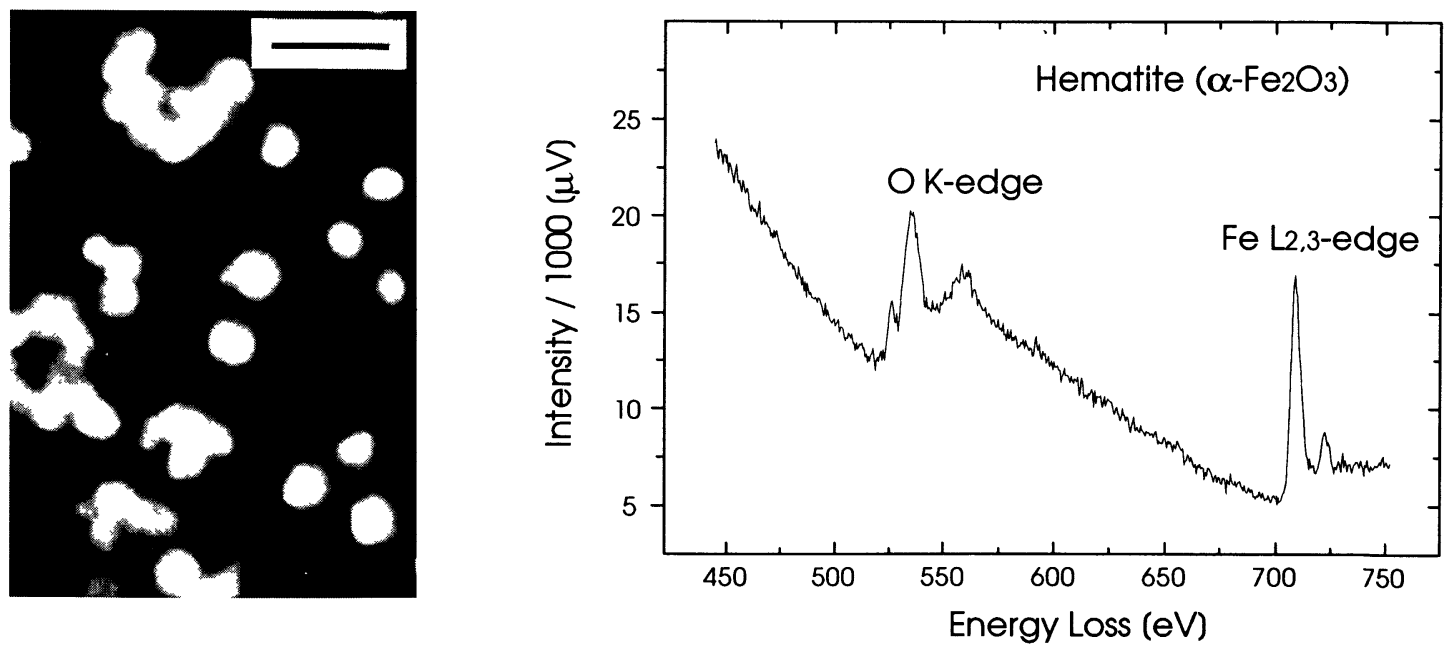

Fig. 2. - Individual suspension of hematite. a) ESI of iron (bar $=200 \mathrm{~nm}$ ); b) EELS recorded on image a). 

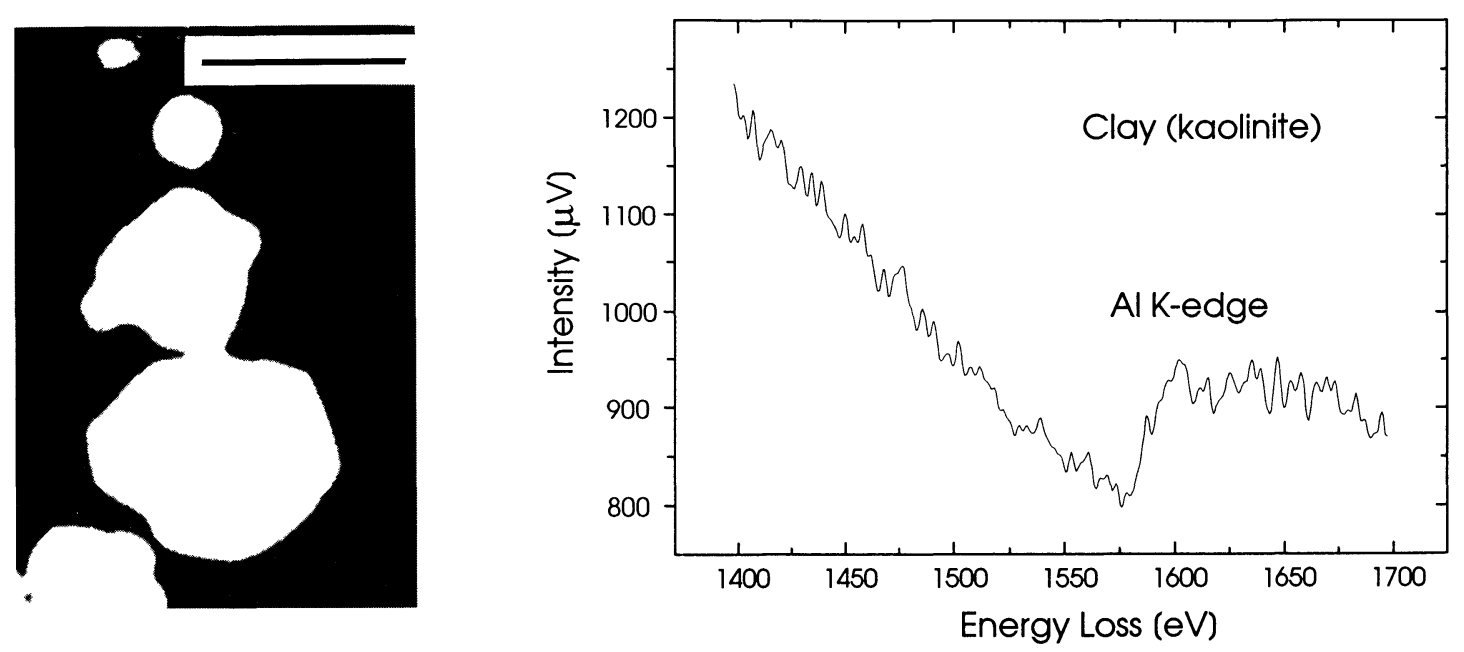

Fig. 3. - Individual suspension of kaolinite. a) ESI of aluminum (bar $=200 \mathrm{~nm})$; b) EELS recorded on image a).
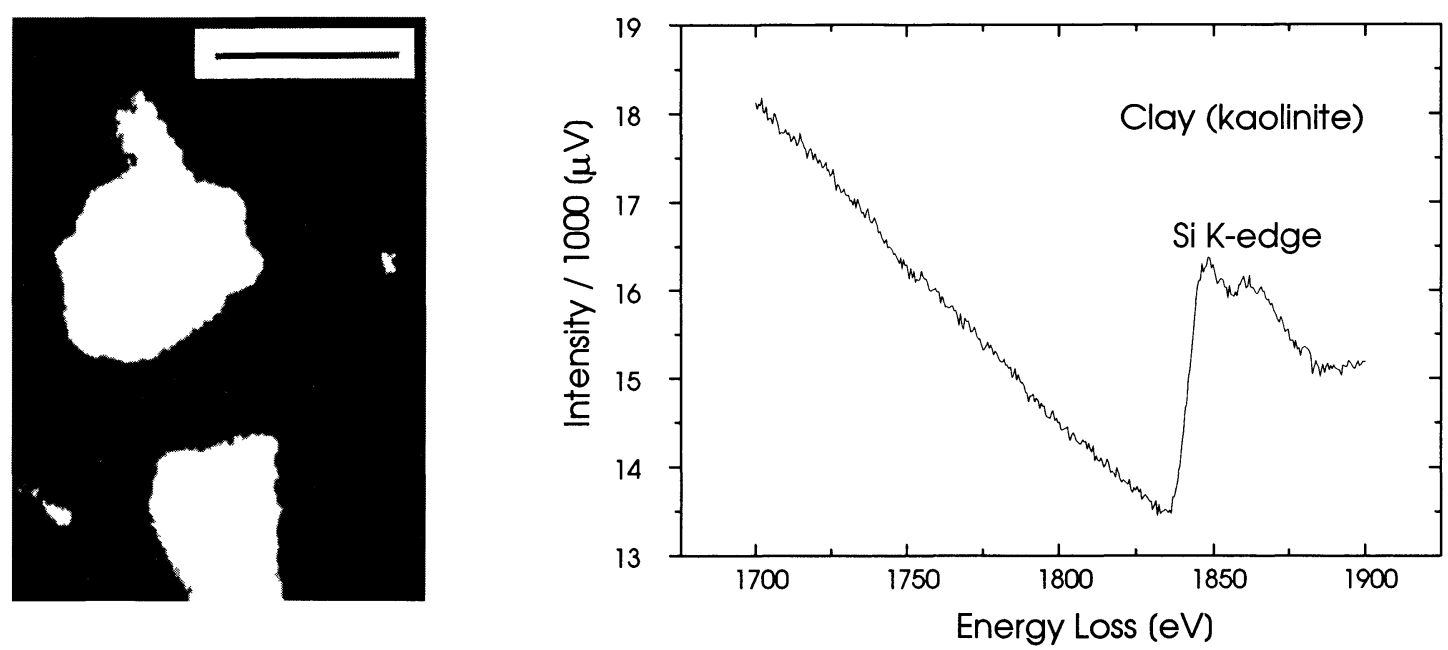

Fig. 4. - Individual suspension of kaolinite. a) ESI of silicon (bar $=200 \mathrm{~nm}$ ); b) EELS recorded on image a).

for background stripping at $1600 \mathrm{eV}$. The $\mathrm{Al} L_{2,3}$ edge can be measured at $70 \mathrm{eV}$; however, this edge rises as a shoulder in the plasmons region and elemental images of $\mathrm{Al}$ from the $L_{2,3}$ edge could lead to biased results produced by incorrect background estimation. As a consequence, the $\mathrm{Al} \mathrm{K}$ edge is prefered for ESI applications, although the signal intensity is relatively low at this energy.

Silicon in kaolinite (Fig. 4b) was recorded with the same energy window (acquisition time: $8 \mathrm{~s} / \mathrm{image})$. Pre-edges at $1790 \mathrm{eV}$ and $1800 \mathrm{eV}$ were used for background extrapolation at $1900 \mathrm{eV}$. 


\section{Results and Discussion}

Figure 5a is an unfiltered bright-field TEM image of an heterogeneous mixture of hematite, kaolinite and alumina with lightly stained xanthan. This image is similar to images obtained on real aquatic submicron particles, thus indicating that our heterogeneous laboratory mixture is a realistic and representative model of natural aquatic suspensions.

As expected, bright-field TEM does not allow visualization of polysaccharide fibrils. This is due to the fact that specimen grids were prepared by successive deposition of $i$ ) hematite + xanthan + uranyl acetate, then ii) aluminum oxide, finally iii) kaolinite. Therefore, the stain associated to fibrils collected on the grid during the first preparation step was partially washed away during the 2 further collection steps.

However, EF-TEM in CT mode at $250 \mathrm{eV}$ (Fig. 5b) reveals thin polysaccharide fibrils (ca. 10 $\mathrm{nm}$, see arrows) present as large scale networks systematically trapping mineral microparticles. From an environmental point of view, this observation is of the utmost importance, as it suggests that polysaccharide fibrils have a strong tendency to assemble into networks and to bridge mineral particles together.

It has to be pointed out that such organic networks, probably polysaccharidic in nature, are not systematically revealed in aquatic specimens by bright-field TEM because of their poor electron density, and that their identification by CT-TEM could increase our knowledge on mineral microparticles/organic macromolecules interactions in natural systems.

Staining of these polysaccharides with a higher concentration of uranyl has been shown to reveal artefacted structures, even by bright-field TEM, because the contrasting agent tends to accumulate at the surface of fibrils, thus artificially thickening them and favoring their coalescence. On the other hand, a concentration of stain $<0.1 \%$ impairs the quality of CT images at the ultrastructural level.

Elemental mapping by ESI and spectrum by EELS of microparticles present in Figure 5a have been acquired under optimum conditions described for the individual suspensions. Figures 5ce are elemental mappings of iron, aluminum and silicon, respectively extracted from Figure 5a in ESI mode. For convenience, ESI of aluminum is a positive-negative inverted image, $\mathrm{Al}$-rich entities appearing darker than their surrounding. These images show that the major constituents of microparticles (in our case $\mathrm{Fe}, \mathrm{Al}, \mathrm{Si}$ ) can be selectively identified within an heterogeneous specimen, at the level of each individual particle.

The combination of Figures 5a, and 5c-e is of special interest for the following reasons. First, iron-rich microparticles (in our case hematite) are clearly identified, even when they are obscured in bright-field TEM by larger entities overlapping them (compare Figs. 5a and 5c). Second, ESI of aluminum indicates that 2 aluminum-rich entities are present in the analysed area, while ESI of silicon reveals only 1 silicon-rich particle (compare Figs. 5d and 5e). From these results, correlated to morphological estimations in Figure 5a, it is clear that the Si/Al-rich particle is a kaolinite microparticle while the $\mathrm{Al}$-rich entity is an aggregate of individual aluminum oxide microparticles.

Tentative characterization of particles with thickness in the $>150 \mathrm{~nm}$ range failed in producing good quality elemental mapping images and could not reveal overlapped particles of different nature. This is most probably due to mass-thickness and multiscattering effects in the bulk of thick particles or aggregates.

Figure 6 shows EEL Spectra which have been recorded separately at high magnification $\left(20^{\prime} 000 \mathrm{x}\right)$ for the various individual hematite, aluminum oxide, respectively kaolinite microparticles present in Figure 5a. These spectra are in good agreement with the corresponding ESI identifications and allow determination of the elemental composition of each analyzed particle.

For the sake of environmental applications, where microparticles have an unknown composition, it would be necessary to record the EEL Spectrum in the $0 \mathrm{eV}$ to $2000 \mathrm{eV}$ range, in order 

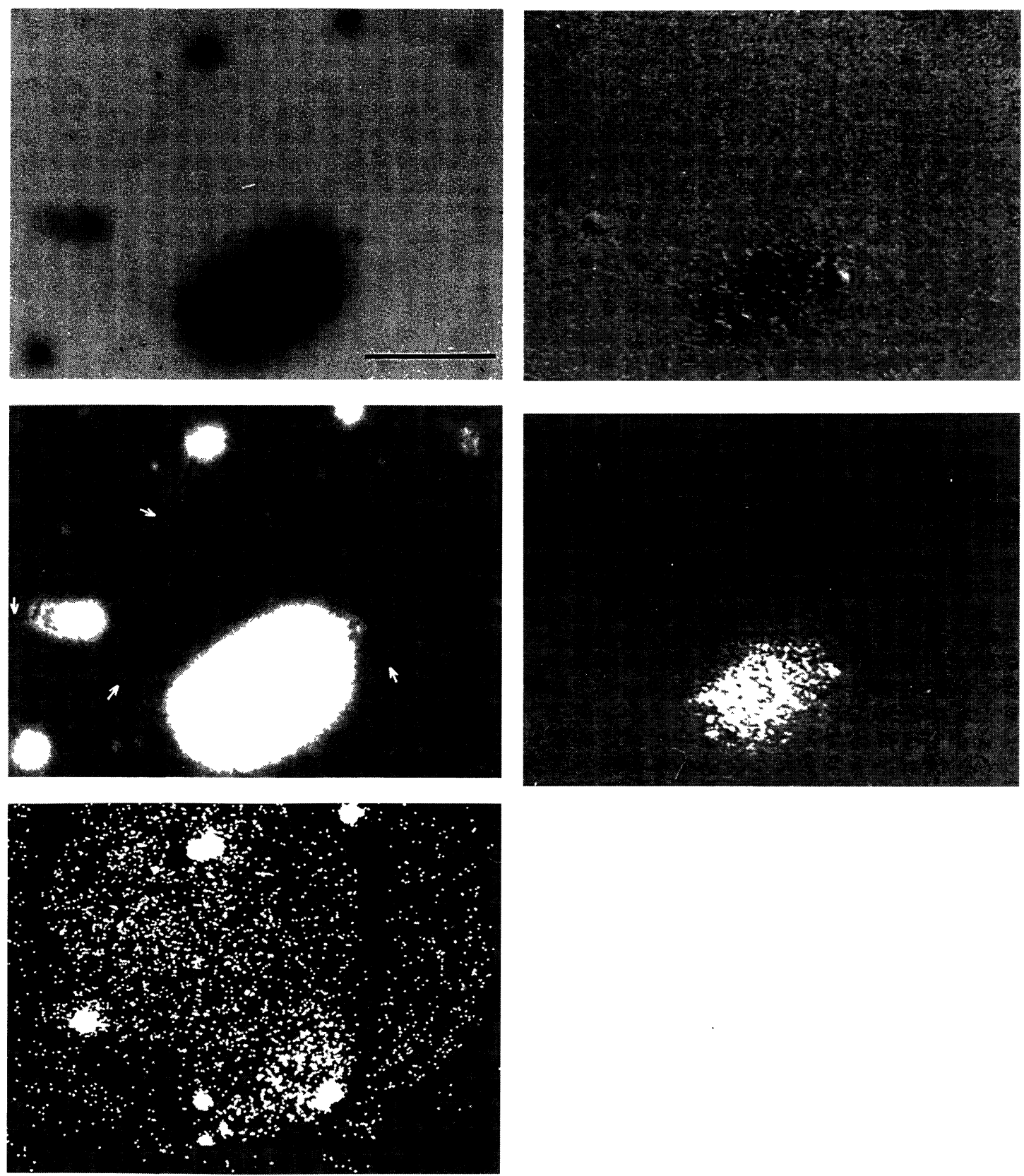

Fig. 5. - Heterogeneous mixture of hematite, aluminum oxide, kaolinite and xanthan. a) Bright field TEM. b) CT image recorded at $\Delta E_{\text {loss }}=250 \mathrm{eV}$, showing xanthan fibrils. c) ESI of iron-containing microparticles (Fe $\left.L_{2,3}\right)$. d) ESI of aluminum-containing microparticles (Al K). e) ESI of silicon-containing microparticles $(\mathrm{Si} \mathrm{K})$. Bar $=500 \mathrm{~nm}$. 

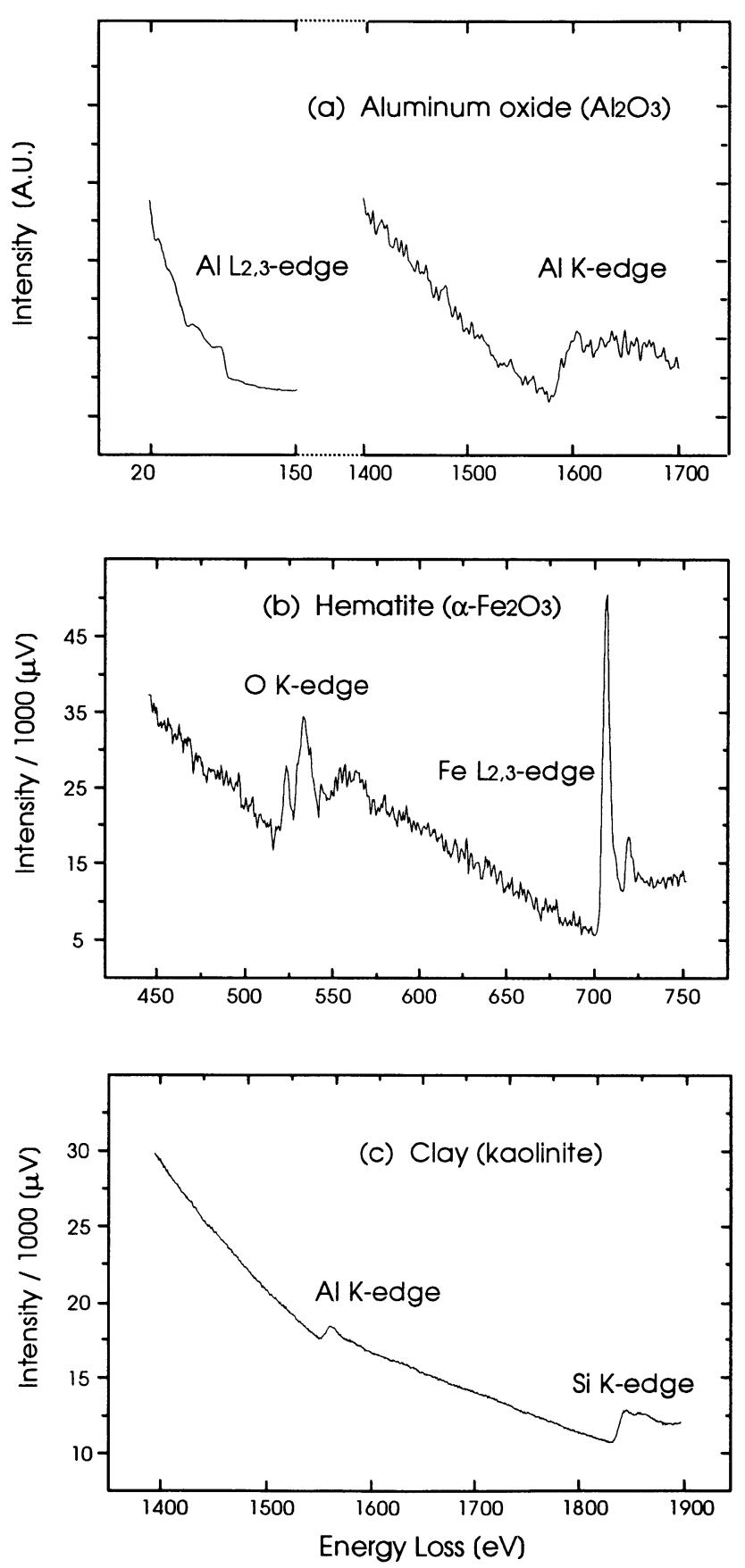

Fig. 6. - EELS fingerprints of a) aluminum oxide, b) hematite and c) kaolinite microparticles present in Figure 5. 
to obtain a complete fingerprint of the composition of the analyzed particle, prior to proceed to elemental mapping by ESI. However, we have experienced that lateral drift occuring during the long spectrum acquisition time (ca. 1h) is a major drawback for serial EELS applications, and we would strongly recommend to use a parallel EEL Spectrometer when unknown fingerprint recording is required.

\section{Conclusions}

Characterization of natural aquatic microparticles has increased in importance during the last few years. Our results show that analytical difficulties linked to these microparticles, mainly their low mass concentration range and their small sizes, can be addressed by Analytical Electron Microscopy in TEM-EELS-ESI modes.

Suspensions containing heterogeneous mixtures of synthetic iron oxide, aluminum oxide and kaolinite in the $<200 \mathrm{~nm}$ range, in the presence of flexible polysaccharide fibrils, adequately mimic complex aquatic samples.

Chemical composition of these model mixtures can be profitably extracted by either Electron Energy Loss Spectrometry or Electron Spectroscopic Imaging. EELS allows fingerprinting and unambiguous identification of the composition of individual microparticles, while ESI allows elemental mapping and can even specifically reveal particles of a given $t$ ype when they are present in relatively large heterogeneous aggregates, although they might be completely obscured in brightfield TEM.

Furthermore, polysaccharide macromolecules, usually electron-translucent in unfiltered images, are clearly contrasted at the ultrastructural level in Contrast Tuning mode, thus revealing their large-scale networking and their influence in the bridging of microparticles.

For microparticles of unknown composition, parallel EELS analysis would greatly enhance capabilities of the method by reducing the time necessary for recording a spectrum over a wide range of energies.

\section{Acknowledgements}

We are grateful to the team of Dr. S. Fakan (Center of Electron Microscopy, University of Lausanne) for help on EM. This work was supported by a grant from the Swiss National Science Foundation (project 21-36593.92).

\section{References}

[1] Buffle J. and Van Leeuwen H.P., Environmental Particles, Vol. 1 (Lewis, Chelsea, 1992).

[2] Buffle J. and Van Leeuwen H.P., Environmental Particles, Vol. 2 (Lewis, Chelsea, 1993).

[3] Perret D., Newman M.E., Nègre J.-C., Chen Y. and Buffle J., Wat. Res. 28 (1994) 91.

[4] Fortin D., Leppard G.G. and Tessier, A., Geochim. Cosmochim. Acta 57 (1993) 4391.

[5] Filella M., Buffle J. and Leppard G.G., Wat. Sci. Tech. 27 (1993) 91.

[6] Wells M.L. and Goldberg E.D., Nature 353 (1991) 342.

[7] Nomizu T., Nagamine J. and Mizuike A., Mikrochim. Acta III (1989) 69.

[8] Leppard G.G., Buffle J., DeVitre R.R. and Perret D., Arch. Hydrobiol. 113 (1988) 405.

[9] Leppard G.G., DeVitre R.R., Perret D. and Buffle J., Sci. Tot. Environ. 87/88 (1989) 345. 
[10] Nomizu T., Goto K. and Mizuike A., Anal. Chem. 60 (1988) 2653.

[11] Degueldre C., Longworth G., Moulin V., Vilks P., Ross C., Bidoglio G., Cremers A., Kim J., Pieri J., Ramsay J., Salbu B. and Vuorinen U., Grimsel Colloid Exercise: an International Intercomparison Exercise on the Sampling and Characterization of Groundwater Colloids (Paul Scherrer Institut, Internal Report TM-36, Würenlingen and Villigen, 1989).

[12] Van Grieken R., Artaxo P., Bernard P., Bruynseels F., Otten P., Storms H. and Xhoffer C., Stud. Environ. Sci. 34 (1988) 307.

[13] Egerton R.F., Electron Energy-Loss Spectroscopy in the Electron Microscope (Plenum Press, New York, 1986).

[14] Door R., Frösch D. and Martin R., J. Microsc. 162 (1991) 3.

[15] Mavrocordatos D., Lienemann C.-P. and Perret D., Mikrochim. Acta 117 (1994) 39.

[16] Reimer L., Fromm l., Hirsch P., Platte U. and Rennekamp R., Ultramicroscopy 46 (1992) 335.

[17] Gregory J., Effects of Polymers on Colloid Stability, in: The Scientific Basis of Flocculation, K.J. Ives Ed. (Sijthoff and Noordhoff, Alphen aan den Rijn, 1978) p. 101.

[18] Lyklema J., How Polymers Adsorb and Affect Colloid Stability, Proceedings of the Engineering Foundation Conference on Flocculation, Sedimentation and Consolidation, B.M. Moudgil and P. Somasoundaran Eds. (NY Engineering Foundation, New York, 1985) p. 3.

[19] Penners N.H.G., The Preparation and Stability of Monodisperse Colloidal Haematite $\left(\alpha-\mathrm{Fe}_{2} \mathrm{O}_{3}\right)(\mathrm{PhD}$ Thesis, University of Wageningen, Wageningen, 1985).

[20] Liang L., Effects of Surface Chemistry on Kinetics of Coagulation of Submicron Iron Oxide Particles $\left(\alpha-\mathrm{Fe}_{2} \mathrm{O}_{3}\right)$ in Water (PhD Thesis, Caltech, Pasadena, 1988).

[21] Matijevic E. and Schneider P., J. Colloid Interface Sci. 63 (1977) 509.

[22] Perret D., Leppard G.G., Müller M., Belzile N., DeVitre R. and Buffle J., Wat. Res. 25 (1991) 1333.

[23] Nomizu T. and Mizuike A., Mikrochim. Acta I (1986) 65.

[24] Ahn C.C., Krivanek O.L., Burgner R.P., Disko M.M. and Swann P.R., EELS Atlas (Gatan, Warrendale, 1983).

[25] Reimer L., Zepke U., Moesch J., Schulze-Hillert S., Ross-Messemer M., Probst W. and Weimer E., EELSpectroscopy: a Reference Handbook of Standard Data for Identification and Interpretation of Electron Energy Loss Spectra and for Generation of Electron Spectroscopic Images (Carl Zeiss, Oberkochen, 1992). 